\title{
Anomalous particle-production thresholds through systematic and non-systematic quantum-gravity effects
}

\author{
Giovanni AMELINO-CAMELIA ${ }^{a}$, Y.Jack NG ${ }^{b}$ and Hendrik VAN DAM ${ }^{b}$ \\ ${ }^{a}$ Dipart. Fisica, Univ. Roma "La Sapienza", P.le Moro 2, 00185 Roma, Italy \\ ${ }^{b}$ Institute of Field Physics, Department of Physics and Astronomy, \\ University of North Carolina, Chapel Hill, NC 27599-3255, USA
}

\begin{abstract}
A growing number of studies is being devoted to the identification of plausible quantum properties of spacetime which might give rise to observably large effects. The literature on this subject is now relatively large, including studies in string theory, loop quantum gravity and noncommutative geometry. It is useful to divide the various proposals into proposals involving a systematic quantum-gravity effect (an effect that would shift the main/average prediction for a given observable quantity) and proposals involving a non-systematic quantumgravity effect (an effect that would introduce new fundamental uncertanties in some observable quantity). The case of quantum-gravity-induced particle-production-threshold anomalies, a much studied example of potentially observable quantum-gravity effect, is here used as an example to illustrate the differences to be expected between systematic and nonsystematic effects.
\end{abstract}




\section{Introduction}

Recently, there has been increasing interest in "Quantum-Gravity Phenomenology" |1, 2, the research programme that attempts to identify aspects of quantum gravity that can be tested experimentally and to identify some of the corresponding experiments that can do significant tests with presently-available technological capabilities. Examples of possible experimental searches have been considered in Refs. [3, 4, 5, 6, 7, 8, 9, 10, 11, 12 and (with an emphasis not directly related to quantum properties of space-time) in Refs. 11, 13]. Recent reviews of the status of this field can be found in Refs. [14, 2.

Here we want to draw a sharper distinction between analyses that consider "systematic" quantum-gravity effects and analyses that consider "non-systematic" quantum-gravity effects. The theoretical prediction that does not include the effects of quantum gravity is characterized, with respect to a given observable $\hat{X}$, by a prediction $X$ and, possibly, a fundamental (quantum mechanical) uncertainty $\delta X$. The effects of quantum gravity in general then should lead [2] to a new prediction $X^{\prime}$ and a new uncertainty $\delta X^{\prime}$. One would attribute to quantum gravity the effects $(\Delta X)_{Q G} \equiv X^{\prime}-X$ and $(\delta X)_{Q G} \equiv \delta X^{\prime}-\delta X \geq 0$. One can

speak of purely systematic quantum-gravity effect when $(\Delta X)_{Q G} \neq 0$ and $(\delta X)_{Q G}=0$, while the opposite case, $(\Delta X)_{Q G}=0$ and $(\delta X)_{Q G}>0$, can be qualified as purely non-systematic. One might expect both types of effect to be present simultaneously, as it is emerging from certain developing formalisms [15], but in a given phenomenological programme the focus may be put on one or the other case. For example, the type of in-vacuo dispersion studies described in Refs. 14, 5] focuses on a purely systematic effect, while the space-time-foam interferometric studies described in Ref. 66, 7, 8] focus on a purely non-systematic effect.

We intend to characterize more carefully the differences between these two types of quantum-gravity effects, even though, depending on the specifics of the physical context, the signatures to be sought experimentally may or may not be sharply different. Our analysis will mainly rely on the case study provided by one of the possible quantum-gravity predictions which has recently attracted most attention: the possibility of quantum-gravity implications for the kinematical threshold for certain particle-physics processes. In that context, which we will review in the next section, both types of effects can be meaningfully considered. We will discuss their different manifestations, and in particular we will argue that the characteristic signatures of nonsystematic effects have a stronger tendency to depend on some nontrivial features of the underlying dynamical theory, whereas in some cases systematic effects can be studied with the exclusive guidance of symmetry principles. These points we will articulate in Section 3, while Section 4 is devoted to some closing remarks.

\section{Quantum-gravity-induced threshold anomalies}

In two different regimes, UHECRs and multi-TeV photons, the universe appears to be more transparent than expected. UHECRs should interact with the Cosmic Microwave Background Radiation (CMBR) and produce pions. TeV photons should interact with the Far Infra Red Background (FIRB) photons and produce electron-positron pairs. These interactions should make observations of UHECRs with $E>5 \cdot 10^{19} \mathrm{eV}$ (the GZK limit) [16] or of gamma-rays with $E>10 \mathrm{TeV} \llbracket 17$ from distant sources unlikely. Still UHECRs above the GZK limit and Mk501 photons with energies up to $24 \mathrm{TeV}$ have been observed.

A CMBR photon and a UHE proton with $E>5 \cdot 10^{19} \mathrm{eV}$ should satisfy the kinematic requirements (threshold) for pion production. UHE protons should therefore loose energy, due to photopion production, and should slow down until their energy is below the GZK energy. At higher energies the proton's mean free path decreases rapidly and it is down to a few Mpc at $3 \cdot 10^{20} \mathrm{eV}$. Yet more than $15 \mathrm{CRs}$ have been observed with nominal energies at or above $10^{20} \pm 30 \% \mathrm{eV} 18 \|$. There are no astrophysical sources capable of accelerating particles to such energies within a few tens of Mpc from us. Furthermore, if the CRs are produced 
homogeneously in space and time, we would expect a break in the CR spectrum around the GZK threshold, which is not seen.

HEGRA has detected high-energy photons with a spectrum ranging up to $24 \mathrm{TeV}$ [19 from Markarian 501 (Mk501), a BL Lac object at a redshift of $0.034(\sim 157 \mathrm{Mpc})$. This observation might indicate a second paradox of a similar nature. A high energy photon with energy $E$ can interact with a FIRB photon with wavelength $\lambda \sim 30 \mu m(E / 10 T e V)$ and produce an electron-positron pair. The mean free path of $\mathrm{TeV}$ photons depends on the spectrum of the FIRB. Recent data from DIRBE[20, 21, 22] and from ISOCOM 23 suggest that the mean free path for $20 \mathrm{TeV}$ photons should be much shorter than the one of $10 \mathrm{TeV}$ photons. However, no apparent break is seen in the spectrum of Mk501 in the region $10-20 \mathrm{TeV}$.

The UHECR paradox is well established. Numerous theoretical models, mostly requiring new physics, have been proposed for its resolution (see Ref. 224 for a recent review). With much less data, and with some uncertainty on the FIRB, the Mk501 TeV-photon paradox is less established If. However, if indeed this must be considered as a paradox, there are no models for its resolution, apart from the possibility that the FIRB estimates are too large. Planned experiments will soon provide us better data on both issues. At present it appears reasonable to explore the possibility that both paradoxes are real.

One natural interpretation of these paradoxes relies on quantum gravity effects [9, 10, 11]: both classes of puzzling observations, cosmic rays and Mk501 photons, at the theoretical level involve the evaluation of a particle production threshold. The relevant threshold conditions are nothing but a statement about the symmetries of classical Minkowski spacetime. It is therefore conceivable that one might explain the puzzling observations in terms of a quantumgravity-induced "threshold anomaly" [10], i.e., a different prediction for thresholds which might result from quantum properties of spacetime. In both paradoxes, low-energy photons interact with high energy particles. The relevant reactions should take place at a kinematic threshold. In both cases, the center-of-mass threshold energies are rather modest and the physical processes involved are well tested and understood. These observations appears to encourage the hypothesis that quantum-gravity-induced deviations from ordinary Lorentz invariance might be responsible for both paradoxes 2 .

Most studies have considered the possibility that quantum-gravity effects might shift the threshold systematically (in the language adopted in the introduction, this would be a "systematic" quantum-gravity effect). In order to illustrate this systematic mechanism of threshold anomalies, let us consider, for example, the case in which quantum-gravity effects are assumed to induce a deformation of the dispersion relations of the type

$$
E^{2} \simeq \vec{p}^{2}+m^{2}+\eta \vec{p}^{2}\left(\frac{E}{E_{p}}\right)^{\alpha}
$$

where $E$ and $\vec{p}$ are the energy and the (3-component) momentum of the particle, $E_{p}$ is the Planck energy scale $\left(E_{p} \sim 10^{16} \mathrm{TeV}\right), \alpha$ and $\eta$ are free parameters characterizing the deviation from ordinary Lorentz invariance. [3

\footnotetext{
${ }^{1}$ For an analysis of the Mk501 experimental situation that does not advocate threshold anomalies see, e.g., Ref. 25].

${ }^{2}$ It is of course also possible to consider deviations from Lorentz invariance that do not have quantumgravity origin [26], but, as discussed in Refs. [10], the idea of a quantum-gravity origin, besides being conceptually appealing, leads to a natural estimate for the magnitude of the effects, and this estimate appears to fit well the observations (while non-quantum-gravity approaches host a large number of parameters which have to be freely adjusted to obtain the needed magnitude of departures from Lorentz-invariance).

${ }^{3}$ Space-time structures leading to (1) do not necessarily reflect a loss of symmetry with respect to ordinary Minkowski space-time. For example, with $\alpha=1$, (1) can characterize certain quantum spacetimes as an
} 
Experimental tests of the predictions of (1) were first proposed in Ref. [4], for the case $\alpha=1$, and in Ref. [1], for generic $\alpha$.

Let us consider the implications of (11) for the kinematics of the process of electronpositron pair production, which is relevant for the Mk501 paradox. Combining (1) with ordinary energy-momentum conservation rules, one can establish that, for the case $\alpha=1$, at threshold the energy $E$ of the hard Mk501 photon and the energy $\epsilon$ of the soft background photon satisfy the relation $E \simeq m_{e}^{2} / \epsilon-\eta m_{e}^{6} /\left(8 E_{p} \epsilon^{4}\right)$. For negative $\eta$ (and $\left.|\eta| \sim 1\right)$, the correction $-\eta m_{e}^{6} /\left(8 E_{p} \epsilon^{4}\right)$ is indeed sufficient to push the threshold energy upwards by a few $\mathrm{TeV}$, consistently with observations. As shown in Refs.]10] (and references therein), an analogous result holds for the photopion threshold, which is relevant for the cosmic-ray paradox.

This type of analysis shows that the two paradoxes might indeed be a manifestation of a quantum (Planck-length related) property of spacetime.

We have so far assumed that the quantum-gravity effect responsible for the puzzling observations in cosmic-ray and Mk-501 physics is of the systematic type, but in Ref. [11] some non-systematic quantum-gravity effects were considered in relation with these same observations. Our main objective here is to compare the non-systematic effects with the already understood systematic effects.

\section{$3 \quad$ Non-systematic effects and threshold anomalies}

In the previous Section, we have reviewed the use of a systematic quantum-gravity effect, a systematically deformed dispersion relation, for the solution of threshold anomalies. One can easily construct an alternative scheme in which the systematic effect responsible for the anomalies appears in energy-momentum conservation (or both in the dispersion relation and in energy-momentum conservation), but we shall not be concerned with these alternatives, since our focus is on distinguishing between systematic and non-systematic effects.

Accordingly, in this Section, we consider the case of a non-systematic quantum-gravityinduced deformation of the dispersion relation, more specifically, the case in which the classical relation $E^{2}=p^{2}+m^{2}$ still holds on average, but in a given physical realization $E$ will be related to $p^{2}$ in a way that can be slightly different from the average relation: $E^{2}=p^{2}+m^{2}+\Delta$, with $-\eta \vec{p}^{2}\left(E / E_{p}\right)^{\alpha}<\Delta<\eta \vec{p}^{2}\left(E / E_{p}\right)^{\alpha}$.

As we shall see, the implications of these non-systematic effects may depend heavily on the mechanism by which the uncertainty (the non-systematic effect) is attributed in a given physical process. In preparation for that discussion it is useful to consider a simplified problem, which captures some aspects of the threshold-anomaly problem here of interest.

\subsection{A useful analogy}

The observation of cosmic-rays or photons above threshold is puzzling because they come from far away and they should have disappeared (or "degraded", in the case of cosmic rays) along the way, by encountering appropriate "sinks" (the soft background photons).

For the purpose of the analysis we are reporting here, it is useful to consider a much simplified analogous problem. Consider a setup in which wheels, of diameter $D_{w}$, are sent from point $A$ to point $B$. On the path from $A$ to $B$, there are some holes of diameter $D_{h}$.

observer-independent property [15], and in those spacetimes one still has a (modified) concept of Lorentz invariance which amounts to as many symmetries as in the ordinary classical-spacetime case. In this Section we are, however, effectively considering a broken-symmetry case; in fact, we assume ordinary conservation of energy-momentum, while the case in which a dispersion relation (1) does not lead to symmetry loss involves [15] a corresponding deformation of energy-momentum conservation. 
If $D_{w}<D_{h}$ the wheels will disappear in the first hole encountered and will not reach point $B$. If $D_{w}>D_{h}$ the wheels will safely reach point $B$.

One can speak of a threshold anomaly for the case in which the classical predictions for $D_{w}$ and $D_{h}$ are such that $D_{w}<D_{h}$, but some wheels still reach point $B$ from $A$. We are assuming that the number of wheels sent from $A$ toward $B$ per unit time is not known. In the case of the "classical" prediction $D_{w}<D_{h}$ the ignorance about this frequency of emission is not significant: in the case $D_{w}<D_{h}$ no wheels should reach $B$. If some wheels are observed at $B$ something must be wrong with the "classical theory".

Such an anomaly could be explained as a systematic "non-classical" effect: one could argue that either the correct (non-classical) value of the wheel diameter $D_{w}^{\prime}$ is such that $D_{w}^{\prime}>D_{h}>D_{w}$ or that the correct value of the hole size $D_{h}^{\prime}$ is such that $D_{h}^{\prime}<D_{w}<D_{h}$ (in other words one should assume that the classical estimate, $D_{w}, D_{h}$, is systematically incorrect, and that the correct values of wheel diameter and the whole size are some $D_{w}^{\prime}, D_{h}^{\prime}$ such that $\left.D_{h}^{\prime}<D_{w}^{\prime}\right)$.

One can also advocate non-systematic effects. Assume for example that, while the classical estimate of the hole size $D_{h}$ is correct, the correct non-classical description of the mechanism that sends wheels from $A$ to $B$ is such that not all wheels have the same diameter $D_{w}$, but any given wheel has a diameter $D_{w}^{\prime}$ with $D_{w}-\Delta<D_{w}^{\prime}<D_{w}+\Delta$, according to some probability law. Then it would not be surprising to observe some wheels arriving at $B$, since some of the wheels do have a diameter $D_{w}^{\prime}$ such that $D_{w}^{\prime}>D_{h}$. This would provide an explanation of the threshold anomaly.t

On the other hand, if the classical estimate of $D_{w}$ is correct but a non-classical uncertainty affects $D_{h}$, one does not necessarily obtain an explanation of the threshold anomaly. The holes found on the way between point $A$ and point $B$ may not all have the same size $D_{h}$, but instead the i-th hole has size $D_{h}^{i}$ with value somewhere in the range $D_{h}-\Delta<D_{h}^{i}<D_{h}+\Delta$, according to some probability distribution. In this case, if only one (or a few) hole is encountered on the way from $A$ to $B$, there will be a significant portion of the wheels that reachs $B$, providing an explanation for the threshold anomaly. But if the number of holes found on the way from $A$ to $B$ is large, then there will only be a negligibly small probability for a wheel to reach point $B$; in fact, out of many holes there will easily be at least one with size $D_{h}^{i}$ such that $D_{w}<D_{h}^{i}$. A non-systematic effect, even one that at first sight might appear well suited for providing an explanation for the threshold anomaly, is not necessarily sufficient to solve the threshold anomaly.

In closing this Subsection, let us consider a scenario in which both systematic and nonsystematic effects coexist. Let us assume that the classical estimate $D_{w}$ of the size of the wheels is incorrect and must be replaced systematically with the estimate $D_{w}^{\prime}$, and that $D_{w}^{\prime}>D_{h}$. Moreover, let us assume that the size of the holes is $D_{h}$ only on average, but the i-th hole has size $D_{h}^{i}$ with value somewhere in the range $D_{h}-\Delta<D_{h}^{i}<D_{h}+\Delta$, according to some probability distribution. In such a situation it is crucial to establish whether $D_{w}^{\prime}$ is greater than $D_{h}+\Delta$, because, in spite of the fluctuations in the sizes of the holes, this would mean that the wheels can get safely from $A$ to $B$. But, if $D_{w}^{\prime}<D_{h}+\Delta$, and the number of holes is large, it is then likely that the wheel will encounter on the way from $A$ to $B$ at least one hole large enough for the wheeel to disappear into it. This scenario is interesting because it shows that, for theories with both systematic and nonsystematic effects, one should really do a careful analysis: it is not sufficient to analyze the systematic effects. We have seen, via this analogy, that non-systematic effects can in some situations give rise to a systematic result (in our analogy, if the number of holes is large the wheel will disappear into one of the holes, nearly systematically). And non-systematic effects can counter-act the action of systematic effects (in our analogy, the systematic effect in the wheel diameter, which would have explained the threshold anomaly, is offset by the non-systematic effect on the sizes of the holes).

\footnotetext{
${ }^{4}$ This is analogous to the non-systematic effect advocated in Ref. [11].
} 


\subsection{Threshold anomalies and non-systematic quantum-gravity ef- fects}

In Section 2 we have reviewed the most common use of quantum-gravity ideas in relation with the threshold anomalies: a systematic quantum-gravity effect on the dispersion relation, which affects the threshold prediction. In Ref. [11] another type of quantum-gravity effects was preliminarily considered in relation with the threshold anomalies: the possibility of a new uncertainty principle between energy and momentum.

Motivation for these new uncertainty principles can be found in Ref. [27, 11]. For the purposes of the present study it is significant that a new uncertainty principle introduces new non-systematic effects in quantum-gravity phenomenology. The analogy drawn in the previous Subsection will help us quickly analyze the possible implications of such non-systematic effects for threshold anomalies.

For simplicity, let us focus on some non-systematic effects that are the natural counterpart for the systematic effects described by Eq. (11) for the case $\alpha=1$. Whereas, according to Eq. (1), the relation between energy and momentum at high energies is systematically deformed to

$$
E \simeq|\vec{p}|+\frac{m^{2}}{2|\vec{p}|}+\frac{\eta}{2} \frac{\vec{p}^{2}}{E_{p}},
$$

in an uncertainty-relation-based scheme for non-systematic quantum-gravity effects one can assume that on average the classical-spacetime relation between energy and momentum is still satisfied, but for a given particle with large momentum $\vec{p}$, energy would be somewhere in the range

$$
|\vec{p}|+\frac{m^{2}}{2|\vec{p}|}-\frac{|\eta|}{2} \frac{\vec{p}^{2}}{E_{p}} \leq E \leq|\vec{p}|+\frac{m^{2}}{2|\vec{p}|}+\frac{|\eta|}{2} \frac{\vec{p}^{2}}{E_{p}},
$$

with some (possibly gaussian) probability distribution. The given quantum-gravity theory will host a fundamental value of $\eta$ (reasonably conjectured to be of order 1 [4, 15]), but each given particle will satisfy a dispersion relation of the type

$$
E \simeq|\vec{p}|+\frac{m^{2}}{2|\vec{p}|}+\frac{\tilde{\eta}}{2} \frac{\vec{p}^{2}}{E_{p}}
$$

with $-|\eta| \leq \tilde{\eta} \leq|\eta|$.

Let us again focus on the example of electro-positron pair production in a head-on photonphoton collision, again assuming that (as relevant for the analysis of Mk501 observations) one of the photons is very hard while the other one is very soft. To leading order, the value of $\tilde{\eta}$ for the given soft photon is not important, only the energy $\epsilon$ of the soft photon is significant. The soft photon can, in leading order, be treated as satisfying a classical dispersion relation. In a quantum-gravity theory predicting such non-systematic effects, the hard photon is mainly characterized by its energy $E$ and its value of $\tilde{\eta}$. In order to establish whether a collision between two such photons can produce an electron-positron pair, one should establish whether, for some admissable values of $\tilde{\eta}_{+}$and $\tilde{\eta}_{-}$(the values of $\tilde{\eta}$ pertaining to the positron and the electron respectively), the conditions for energymomentum conservation can be satisfied. The process will be allowed if

$$
E \geq \frac{m^{2}}{\epsilon}-\frac{\tilde{\eta}}{4} \frac{E^{3}}{\epsilon E_{p}}+\frac{\tilde{\eta}_{+}+\tilde{\eta}_{-}}{16} \frac{E^{3}}{\epsilon E_{p}} .
$$

Since $\tilde{\eta}, \tilde{\eta}_{+}$and $\tilde{\eta}_{-}$are bound to the range between $-|\eta|$ and $|\eta|$, the process is only allowed, independently of the value of $\tilde{\eta}$, if the condition

$$
E \geq \frac{m^{2}}{\epsilon}-\frac{3|\eta|}{8} \frac{E^{3}}{\epsilon E_{p}}
$$


is satisfied. This condition defines the actual threshold in the non-systematic-effect scenario we are considering. Clearly in this sense the threshold is inevitably decreased by the nonsystematic effect. However, there is only a tiny chance that a given photon would have $\tilde{\eta}=|\eta|$, since this is the limiting case of the range allowed by the uncertainty principle that is inducing the non-systematic effect, and unless $\tilde{\eta}=|\eta|$ the process will still not be allowed even if

$$
E \simeq \frac{m^{2}}{\epsilon}-\frac{3|\eta|}{8} \frac{E^{3}}{\epsilon E_{p}} .
$$

Moreover, even assuming $\tilde{\eta}=|\eta|$, the energy value described by ( 7 ) will only be sufficient to create an electron positron pair with $\tilde{\eta}_{+}=-|\eta|$ and $\tilde{\eta}_{-}=-|\eta|$, which again are isolated points at the extremes of the relevant probability distributions. Therefore the process becomes possible at the energy level described by (7) but it remains extremely unlikely, strongly suppressed by the small probability that the values of $\tilde{\eta}, \tilde{\eta}_{+}$and $\tilde{\eta}_{-}$would satisfy the kinematical requirements.

With reasoning of this type, one can easily develop an intuition for the dependence on the energy $E$, for fixed value of $\epsilon$ (and treating $\tilde{\eta}, \tilde{\eta}_{+}$and $\tilde{\eta}_{-}$as totally unknown), of the likelihood that the pair-production process can occur: (i) when (6) is not satisfied the process is not allowed; (ii) as the value of $E$ is increased above the value described by (7), pair production becomes less and less suppressed by the relevant probability distributions for $\tilde{\eta}, \tilde{\eta}_{+}$and $\tilde{\eta}_{-}$, but some suppression remains up to the value of $E$ that satisfies

$$
E \simeq \frac{m^{2}}{\epsilon}+\frac{3|\eta|}{8} \frac{E^{3}}{\epsilon E_{p}}
$$

(iii) finally for energies $E$ higher than the one described by (8), the process is kinematically allowed for all values of $\tilde{\eta}, \tilde{\eta}_{+}$and $\tilde{\eta}_{-}$, and therefore the likelihood of the process is just the same as in the classical-spacetime theory.

Up to this point we have analyzed a single photon-photon collision, focusing on the kinematic requirements for electron-positron pair production. This process is relevant for understanding certain astrophysical observations, such as the Mk501 photons. For a Mk501 photon with energy of some 10 or $20 \mathrm{TeV}$, there are many opportunities to collide with soft photons with energy suitable for pair production to occur (the mean free path is much shorter than the distance between the source and the Earth). Thus one expects (in the same sense illustrated through the analogy discussed in the previous Subsection for the case of many holes between points $A$ and $B$ ) that even a small probability of producing an electron-positron pair in a single collision would be sufficient to lead to the disappearance of the MK501 hard photon before reaching our detectors. The probability is small in a single collision with a soft background photon, but the fact that there are, during the long journey, many such pair-production opportunities renders it likely that in one of the many collisions the hard photon would indeed disappear into an electron-positron pair. For the specific scheme of non-systematic effects which we have used as illustrative example in this Subsection, it appears therefore that a characteristic prediction is that the Mk501 should start being sharply suppressed already at the energy level described by ([), below the threshold corresponding to the classical-spacetime kinematics. Of course, completely analogous arguments apply to the analysis of ultra-high-energy cosmic rays. Since the present interpretation of these observations is that the particle flux is unsuppressed even beyond the classical-spacetime prediction, the type of non-systematic scheme considered here appears not to provide an explanation of the data. This of course does not exclude altogether the idea that nonsystematic effects might be responsible for those observations: the non-systematic scheme considered in this Subsection corresponds, in the analogy of the preceding Subsection, only to the case in which non-systematic effects affect the sizes of the many holes between points $A$ and $B$, while the wheel diameter is fixed; but one could look for schemes in which other 
types of non-systematic effects are present. If one could replicate the situation which, in our analogy, involves fixed sizes for the holes but varying diameters for the wheels affected by non-systematic effects, the phenomenological implications for Mk501 observations (and cosmic-ray observations) would be different: one would still expect a suppression of the flux to start occurring below the classical threshold, albeit only a small suppression (which could fall within the accuracy of present measurements), and the flux would then be expected to extend even beyong the classical threshold, although with increasingly large suppression. In this sense, such an alternative non-systematic-effect scheme would provide a solution of the threshold paradoxes.

\subsection{Interplay between systematic and non-systematic effects}

Our analysis of the example of non-systematic effects on particle kinematics may also be relevant for certain systematic-effect schemes for solving the threshold paradoxes. In fact, it appears plausible [15] that in quantum spacetime one would find deformed symmetries, possibly leading to a systematic deformation of the dispersion relation, as well as new uncertainty principles, possibly affecting in non-systematic fashion the $E \leftrightarrow p$ relation established by the dispersion relation. It is therefore important to understand the implications of nonsystematic effects, and in particular we should try to understand how systematic effects and nonsystematic effects can combine in physical contexts such as the ones pertaining to the paradoxes we have considered.

As discussed in the analogy introduced in Subsection 3.1, nonsystematic effects can effectively counter-act the implications of systematic effects of comparable magnitude. Following the reasoning described in Subsection 3.1, the reader can easily realize that it is plausible that one can have a theory in which spacetime symmetries would be such that the dispersion relation is deformed in the direction of inducing an increase of the threshold with respect to the classical-spacetime prediction, but then there might also be non-systematic effects that (with however small probability) can render particle-production processes possible even below the new threshold.

A systematic effect may well raise the threshold for a particle-production process, but in the presence of an accompanying non-systematic effect, this increase of the threshold will have to be interpreted only in a statistical sense: processes with energetics below the new higher threshold can still (with however small probability) occur. If the size of the upward shift in the threshold is comparable to the size of the uncertainties to be taken into account in threshold analysis, the minimum required energetic configuration required by the process will be basically unchanged; the only net implication of the two effects combined would be a probabilistic modulation (of the type discussed in the previous Subsections) of the particle-production probability. When many collision opportunities are encountered by a high-energy particle on its way from the source to the Earth, even a small probability of particle production in each collision may be sufficient for particles below threshold to be involved in at least one particle-production process, and this can counter-act the upward shift of the threshold induced by the systematic effects.

\section{Closing remarks}

The illustrative example of quantum-gravity-induced threshold anomalies has allowed us to show that the analysis of non-systematic quantum-gravity effects can be non-trivial and neglecting such effects in favour of accompanying systematic effects may lead to unreliable conclusions. The stochastic/probabilistic nature of the effects induced by quantum-gravity uncertainty principles can be strengthened in some cases by the fact that several such probabilistic imprints can characterize a given observation. For example, in certain models the probability of particle-production below threshold may be extremely small, but in certain 
situations, as in the case of high-energy particles reaching us from far away astrophysical sources, this small probability applies only to a single opportunity of collision with a soft background photon, while the overall probability of particle production, considering that a large number of particle-production opportunities are encountered by the high-energy particle on its way to our detectors, may still be large.

In light of our analysis, it appears that attempts to explain the debated threshold anomalies for cosmic rays and Mk501 photons that rely exclusively on non-systematic effects are probably problematic; but this type of solution cannot be excluded in general a priori, as the careful reader will deduce from some of the remarks in Subsection 3.1. We have also provided motivations for a more careful study of threshold anomalies in contexts in which both systematic and non-systematic effects are expected, especially since it is not always possible to assume that non-systematic effects are negligible with respect to systematic ones.

\section{Acknowledgments}

G.A.-C. greatfully acknowledges conversations with Tsvi Piran. This work was supported in part by the U.S. Department of Energy and the Bahnson Fund of the University of North Carolina.

\section{References}

[1] G. Amelino-Camelia, "Are we at the dawn of quantum-gravity phenomenology?", grqc/9910089, Lect. Notes Phys. 541, 1-49 (2000).

[2] G. Amelino-Camelia, gr-qc/0012049, Nature 408, 661 (2000); G. Amelino-Camelia, "Quantum-Gravity Phenomenology: Status and Prospects", gr-qc/0204051 (invited Brief Review to appear in a special issue of Modern Physics Letters A devoted to the First IUCAA Meeting on the Interface of Gravitational and Quantum Realms).

[3] J. Ellis, J.S. Hagelin, D.V. Nanopoulos and M. Srednicki, Nucl. Phys. B241, 381 (1984); P. Huet and M.E. Peskin, Nucl. Phys. B434, 3 (1995); V.A. Kostelecky and R. Potting, Phys. Rev. D51, 3923 (1995); O. Bertolami, D. Colladay, V.A. Kostelecky and R. Potting, Phys.Lett. B395, 178 (1997); D.V. Ahluwalia, Mod. Phys. Lett. A13, 2249 (1998).

[4] G. Amelino-Camelia, J. Ellis, N.E. Mavromatos, D.V. Nanopoulos and S. Sarkar, astroph/9712103, Nature 393, 763 (1998).

[5] S.D. Biller et al., Phys. Rev. Lett. 83, 2108 (1999).

[6] G. Amelino-Camelia, gr-qc/9808029, Nature 398, 216 (1999): gr-qc/0104086, Nature 410, 1065 (2001); gr-qc/9903080, Phys. Rev. D62, 024015 (2000).

[7] Y.J. Ng and H. van Dam, gr-qc/9906003, Y.J. Ng and H. van Dam, Found. Phys. 30, 795 (2000); gr-qc/9911054, Phys. Lett. B477, 429 (2000).

[8] G. Amelino-Camelia, gr-qc/0104005.

[9] T. Kifune, Astrophys. J. Lett. 518, L21 (1999); W. Kluzniak, Astropart. Phys. 11, 117 (1999); R.J. Protheroe and H. Meyer, Phys. Lett. B493, 1 (2000).

[10] G. Amelino-Camelia and T. Piran, Phys. Rev. D64, 036005 (2001). 
[11] Y.J. Ng, D.S. Lee, M.C. Oh, and H. van Dam, Phys. Lett. B507, 236 (2001).

[12] G. Amelino-Camelia, gr-qc/0107086, Phys. Lett. B528, 181 (2002).

[13] R. Brustein, gr-qc/9810063; G. Veneziano, hep-th/9902097; M. Gasperini, hepth/9907067; I.C. Percival and W.T. Strunz, Proc. R. Soc. A453, 431 (1997); L.J. Garay, Phys. Rev. Lett. 80, 2508 (1998); D.V. Ahluwalia, gr-qc/9903074. Nature 398, 199 (1999); C. Lämmerzahl and C.J. Bordé, Lect. Notes Phys. 562, 463 (2001).

[14] C. Rovelli, gr-qc/0006061 (in Procedings of the 9th Marcel Grossmann Meeting on Recent Developments in Theoretical and Experimental General Relativity, Gravitation and Relativistic Field Theories, Rome, Italy, 2-9 Jul 2000). S. Carlip, gr-qc/0108040, Rept. Prog. Phys. 64, 885 (2001).

[15] G. Amelino-Camelia, gr-qc/0012051, Int. J. Mod. Phys. D11, 35 (2002); hep-th/0012238, Phys. Lett. B510, 255 (2001); G. Amelino-Camelia, gr-qc/0106004 (in "Karpacz 2001, New developments in fundamental interaction theories", pages 137-150).

[16] K. Greisen, Phys. Rev. Lett. 16, 748 (1966); G. T. Zatsepin and V. A. Kuzmin, Sov. Phys.-JETP Lett. 4, 78 (1966).

[17] A.I. Nikishov, Sov. Phys. JETP 14, 393 (1962); J. Gould, G. Schreder, Phys. Rev. 155, 1404 (1967); F.W. Stecker, O.C. De Jager and M.H. Salomon, Ap.J. 390, L49 (1992).

[18] M. Takeda et al., Phys. Rev. Lett. 81, 1163 (1998); A. Watson, Proc. Snowmass Workshop, p 126, 1996.

[19] F.A. Aharonian et al., A\&A 349, 11A (1999).

[20] E.L. Wright, astro-ph/0004192.

[21] D.P. Finkbeiner, M. Davis and D.J. Schlegel, astro-ph/0004175.

[22] M.G.Hauser, R.G. Arendt, T. Kelsall et al., ApJ 508, 25 (1998).

[23] A. Biviano et al., astro-ph/9910314.

[24] A.V. Olinto, Phys. Rept. 333, 329 (2000).

[25] O.C. De Jager and F.W. Stecker, astro-ph/0107103, Astrophys.J. 566 (2002) 738.

[26] L. Gonzalez-Mestres, physics/9704017; S. Coleman, S.L. Glashow, Phys. Rev. D59, 116008 (1999).

[27] T. Padmanabhan, Class. Quantum Grav. 4, L107 (1987); D.V. Ahluwalia, Phys. Lett. B339, 301 (1994); Y.J. Ng and H. van Dam, Mod. Phys. Lett A9, 335 (1994); G. Amelino-Camelia, Mod. Phys. Lett. A9, 3415 (1994); Y.J. Ng and H. van Dam, Mod. Phys. Lett. A10, 2801 (1995); G. Amelino-Camelia, Mod. Phys. Lett. A11, 1411 (1996). 